\title{
The Relationship between Psychosocial Factors and Periodontal Disease
}

\author{
Cayci E and Guzeldemir-Akcakanat E* \\ Faculty of Dentistry, Department of Periodontology, Kocaeli University, Kocaeli, Turkey
}

\begin{abstract}
Periodontal diseases are inflammatory conditions and many forms of the disease are associated with specific pathogenic bacteria, which colonize the subgingival area. However, the presence of bacteria itself is not capable of producing advanced tissue destruction in all individuals. Initiation and progression of periodontal infections are modified by local and systemic conditions, which defined as risk factors. Systemic risk factors include diabetes mellitus, smoking, age and genetic factors. Recent studies also pointed out to several potentially important risk indicators such as psychosocial factors; stress, depression and ineffective coping, state and trait anxiety. The aim of the present paper is to review the association between psychosocial factors and periodontal disease.
\end{abstract}

Keywords: Periodontal disease; Stress; Depression; Coping; Anxiety

Abbreviations: CRH: Corticotropin-Releasing Hormone; ACTH: Adrenocorticotropic Hormone; IL: Interleukin; TNF- $\alpha$ : Tumor Necrosis Factor Alpha; IFN- $\gamma$ : Interferon Gamma; GCF: Gingival Crevicular Fluid

\section{Review}

Chronic inflammatory periodontal diseases have several etiological factors [1]. Microorganisms in dental plaque biofilms are etiological factors essential for the initiation of the inflammatory process locally at the infection site, and the absence of these, the inflammatory response does not taken place [2]. The presence of bacteria leads the host response which is normally intended to fend the harmful effect of microorganisms. Therefore, an exacerbated or excessive reaction of the host tissue, as well as lack or deficiency of a suitable reaction by the host, may cause or promote host tissue damage [2]. There are several other factors that may also contribute to modulate this interaction, by either potentiating or decreasing the tissue damage. These latter factors include local factors within the mouth, systemic factors related to the host, and environmental factors [2]. Psychosocial factors such as stress, distress, state and trait anxiety, depression and inadequate coping mechanisms are counted as risk factors for periodontal disease and the patterns of the relationship between them are still being investigated.

Several studies have conducted for many years to evaluate the association between psychosocial factors and periodontal disease [3]. In 1962, Davis and Jenkins attempted to determine the correlation between psychological measures of stress and periodontal disease in the human [4].They concluded that there was a significant association between periodontal disease as measured by periodontal index scores, and mental stress. In a preliminary study [5], a total of 10 women and 8 men from the head office of a large company and with a mean age of 39 years were examined at the first visit and over a 12-month period for plaque, bleeding, calculus and pocket depth. Their occupational stress was assessed at the first visit and each subsequent visit. Significant increases in mean plaque score, subgingival calculus, bleeding on probing and pocket depth between weeks 0 and 52 were demonstrated. Including to the authors, the results suggested that susceptibility to periodontal disease might be related to psychological factors, specifically the personality of the individual, which affects reactions to stressful life events including those experienced at work. Linden et al. [6], also assessed the association between occupational stress and the progression of periodontitis and found similar results with the previous study.

Monteiro da Silva et al. [7] examined psychosocial variables in subjects with generalized aggressive periodontitis, chronic periodontitis and periodontally healthy controls. Subjects with generalized aggressive periodontitis had significantly higher self-reported depression and loneliness compared to the chronic periodontitis and control groups. Moss et al. [8] found the influence of social strain, depression and coping on chronic periodontitis. Subgingival plaque was collected and screened for specific microorganisms via immunofluorescence. Subjects with periodontitis were more likely to have high scores on job strain, financial strain, and role strain (e.g. parent, spouse or employee) compared to controls.

Salviet al. [9] considered psychosocial stress as a potential risk factor for periodontal disease according to the studies conducted until that date. Genco et al. [10] correlated various parameters of periodontal diseases with measures of psychological stress, distress and coping behaviors using an age adjusted model in which gender, smoking, diabetes mellitus, T. forsythia and $P$. gingivalis were also included as significant risk indicators. The authors concluded that the effects of stress on periodontal diseases can be moderated by adequate coping behaviors. They further concluded that psychosocial measures of stress associated with financial strain and distress are significant risk indicators for severe periodontal disease in adults.

In a cross-sectional study [11], the investigators researched the prevelance of some negative life events and psychological factors and their relation to periodontal disease. According to them, traumatic life events such as the loss of a spouse may increase the risk for periodontal disease. An individual's coping behavior may play a role in the progression of periodontal disease. Wimmer et al. [12] aimed to determine whether patients with periodontitis have specific stresscoping strategies and whether they differ from those of periodontally healthy controls. They found that periodontitis patients with inadequate stress-behavior strategies are at greater risk for severe periodontal disease.

One of the possible mechanisms of influence of psychosocial

*Corresponding author: Dr. Esra Güzeldemır-Akçakanat, Faculty of Dentistry, Department of Periodontology, Kocaeli University, Kocaeli, Turkey, Tel: 0262344 2222; Fax: 0262344 2109; E-mail: esragd@yahoo.com

Received December 23, 2013; Accepted March 04, 2014; Published March 06 2014

Citation: Cayci E, Guzeldemir-Akcakanat E (2014) The Relationship between Psychosocial Factors and Periodontal Disease. Dentistry 4: 223. doi:10.4172/21611122.1000223

Copyright: ( 2014 Cayci E, et al. This is an open-access article distributed under the terms of the Creative Commons Attribution License, which permits unrestricted use, distribution, and reproduction in any medium, provided the original author and source are credited. 
factors on periodontal health is the alterations of patient's health behavior. Individuals with high stress levels and depression tend to adopt habits which are harmful to periodontal health, such as smoking and alcohol consumption, disruption of sleep patterns, neglecting oral hygiene and poor compliance, bruxism or teeth grinding and nail biting [13]. Besides, the excessive consumption of oily nutrients may cause increased levels of circulating cortisol, thus suppression of the immune system and progression of periodontitis $[14,15]$.

Croucher et al. [16] used a case-control study design to evaluate whether a comprehensive range of life-events were associated with an objective measure of periodontitis in adults. They collected behavioral data including frequencies of tooth brushing and dental attendance and also smoking. They concluded that psychosocial factors and oral health behaviors cluster together as important determinants of periodontitis.

In a cross-sectional study [17], the investigators showed that participants who experienced more stressors, had more clinical attachment loss, missing teeth and deep periodontal pockets. They also found that there was a negative correlation between total stress scores and brushing frequency.

Another mechanism is based on the neuro-immune endocrine interaction by the action of hormones and chemical mediators produced by the organism in situations of stress and anxiety [15]. With stimulation, stress-related homeostasis is maintained by glucocorticoids and catecholamines [18]. Developing physiological response against psychosocial factors leads the stimulation of the autonomic nervous system. Subsequently, the secretion of catecholamines such as epinephrine and norepinephrine occurs. Catecholamines stimulate the formation and activity of prostoglandines and proteolytic enzymes, which can directly provoke tissue destruction. Also, catecholamines lead alterations in the function of immune system cells and increase of blood glucose levels [14]. Activation of the immune system, associated with increases in levels of circulating cytokins, stimulates the hypothalamicpituitary-adrenal axis [19]. When stress is detected by the brain, the central nervous system gets activated and stimulates the hypotalamus to produce corticotropin-releasing hormone (CRH). CRH stimulates the pituitary gland to release adrenocorticotropic hormone (ACTH). ACTH provides the release of glucocorticoids from adrenal cortex [18]. Glucocorticoids can induce the reduction of pro-inflammatory cytokines secretion such as interleukin-1 (IL-1), IL-2, IL-3, IL-6, tumor necrosis factor alpha (TNF- $\alpha$ ), interferon gamma (IFN- $\gamma$ ) [10]. Hence, depressed immune responsiveness occurs and may lead destructive periodontal diseases [9].

Hilgert et al. [19] evaluated the extent and the severity of chronic periodontitis and its association with the levels of salivary cortisol and the scores of an inventory which focuses on stress symptoms in a population aged 50 years and older. The results of this cross-sectional study suggest positive association between cortisol levels and the extent and severity of periodontitis after adjustment for several important variables such as age, sex, oral hygiene, bleeding on probing, smoking and inventory scores.

Johannsen et al. [20] compared oral health status, proinflammatory markers, and cortisol in gingival crevicular fluid (GCF) and saliva in patients with stress-related mental depression and controls to investigate the importance of stress for the development of periodontitis. They found that the levels of cortisol and IL-6 in GCF were significantly higher in patients than in controls.

With a few notable exceptions $[8,10,15]$, many of the reports that investigate the relationship between psychosocial factors and periodontal disease are preliminary and exploratory investigations and use small sample sizes [21-23]. Such small sample studies may be statistically underpowered to detect hypothesized relationships among psychosocial factors and indicators of disease [18]. The criteria used to classify periodontal disease differ from one study to another The variety of methodology applied in existing studies, as well as the absence of a control group and lack of control for confounding variables for periodontal disease, may explain the differences in the results of these studies [21]. In order to obtain accurate results while executing researches, all other factors that may cause periodontal disease or have that potential, should be taken into account. According to Vettoreet al. [23], other potential risk factors such as levels of dental plaque, diabetes mellitus or smoking should be considered when analyzing results to differentiate these factors' possible effects on periodontal status.

\section{Conclusion}

Considering all of the data, future periodontal studies should include prospective, experimental designs with larger sample sizes. Considering the higher degrees of exposition to the psychological factors, psychiatric populations might be chosen in order to establish the association of periodontal disease and the investigated psychosocial factors. The indices that positively identify the disease and the threshold scores should be determined universally, because these differences may limit the comparisons between the investigations. To avoid the misunderstandings when answering the questions, instead of self-reported tests, structured interviews on groups with operational diagnosis might be chosen while determining the subjects' psychological conditions. Finally, the factors which may affect the results such as other risk factors for periodontal disease, should be equalized in case-control studies.

\section{References}

1. Dentino A, Lee S, Mailhot J, Hefti AF (2013) Principles of periodontology Periodontology 2000 61: 16-53

2. Albandar JM (2002) Global risk factors and risk indicators for periodontal diseases. Periodontology 2000 29: 177-206.

3. Peruzzo DC, Benatti BB, Ambrosano GMB, Nogueira-Filho GR, Sallum EA, et al. (2007) A systematic review of stress and psychological factors as possible risk factors for periodontal disease. J Periodontol 78: 1491-1504.

4. Davis CH, Jenkins CD (1962) Mental stress and oral diseases. J Dent Res 41 1045-1049.

5. Freeman R, Goss S (1993) Stress measures as predictors of periodontal disease-a preliminary communication. Community Dent Oral Epidemiol 21 : 176-177.

6. Linden GJ, Mullally BH, Freeman R (1996) Stress and the progression of periodontal disease. J Clin Periodontol 23: 675-680.

7. Monteiro da Silva AM, Oakley DA, Newman HN, Nohl FS, Lloyd HM (1996) Psychosocial factors and adult onset rapidly progressive periodontitis. J Clin Periodontol 23: 789-794

8. Moss ME, Beck JD, Kaplan BH, Offenbacher S, Weintraub JA, et al. (1996) Exploratory case-control analysis of psychosocial factors and adult periodontitis. J Periodontol 67: 1060-1069.

9. Salvi GE, Lawrence HP, Offenbacher S, Beck JD (1997) Influence of risk factors on the pathogenesis of periodontitis. Periodontology 2000 14: 173-201.

10. Genco RJ, Ho AW, Grossi SG, Dunford RG, Tedesco LA (1999) Relationship of stress, distress, and inadequate coping behaviors to periodontal disease. $J$ Periodontol 70: 711-723.

11. Hugoson A, Ljungquist $B$, Breivik $T$ (2002) The relationship of some negative events and psychological factors to periodontal disease in an adult Swedish population 50 to 80 years of age. J Clin Periodontol 29: 247-253.

12. Wimmer G, Janda M, Wieselmann-Penkner K, Jakse N, Polansky R, et al (2002) Coping with stress: its influence on periodontal disease. J Periodonto 73: $1343-1351$. 
Citation: Cayci E, Guzeldemir-Akcakanat E (2014) The Relationship between Psychosocial Factors and Periodontal Disease. Dentistry 4: 223. doi:10.4172/2161-1122.1000223

13. Miller SC, Firestone JM (1947) Psychosomatic factors in the etiology of periodontal disease. J Dent Res 26: 173.

14. Monteiro da Silva AM, Newman HN, Oakley DA (1995) Psychosocial factors in inflammatory periodontal diseases. A review. J Clin Periodontol 22: 516-526.

15. Genco RJ, Ho AW, Kopman J, Grossi SG, Dunford RG, et al. (1998) Models to evaluate the role of stress in periodontal disease. Ann Periodontol 3: 288-302.

16. Croucher R, Marcenes WS, Torres MC, Hughes F, Sheiham A (1997) The relationship between life-events and periodontitis. A case-control study. J Clin Periodontol 24: 39-43.

17. Rosania AE, Low KG, McCormick CM, Rosania DA (2009) Stress, depression, cortisol and periodontal disease. J Periodontol 80: 260-266.

18. LeResche L, Dworkin SF (2002) The role of stress in inflammatory disease, including periodontal disease; review of concepts and current findings. Periodontology 2000 30: 91-103.
19. Hilgert JB, Hugo FN, Bandeira DR, Bozzetti MC (2006) Stress, cortisol and periodontitis in a population aged 50 years and over. J Dent Res 85: 324-328.

20. Johannsen A, Rylander G, Söder B, Asberg M (2006) Dental plaque, gingival inflammation and elevated levels of interleukin- 6 and cortisol in gingival crevicular fluid from women with stress-related depression and exhaustion. J Periodontol 77: 1403-1409.

21. Castro GDC, Oppermann RV, Haas AN, Winter R, Alchieri JC (2006)Association between psychosocial factors and periodontitis: a case-control study. J Clin Periodontol 33: 109-114

22. Solis ACO, Lotufo RFM, Pannuti CM, Brunheiro EC, Marques AH, et al. (2004) Association of periodontal disease to anxiety and depression symptoms, and psychosocial stress factors. J Clin Periodontol 31: 633-638.

23. Vettore MV, Leão AT, Monteiro Da Silva AM, Quintanilha RS, Lamarca GA (2003) The relationship of stress and anxiety with chronic periodontitis. J Clin Periodontol 30: 394-402. 